Delft University of Technology

\title{
Color-Based Proprioception of Soft Actuators Interacting with Objects
}

Scharff, Rob B.N.; Doornbusch, Rens M.; Doubrovski, Eugeni L.; Wu, Jun; Geraedts, Jo M.P.; Wang, Charlie C.L.

DOI

10.1109/TMECH.2019.2929818

Publication date

2019

Document Version

Accepted author manuscript

Published in

IEEE/ASME Transactions on Mechatronics

\section{Citation (APA)}

Scharff, R. B. N., Doornbusch, R. M., Doubrovski, E. L., Wu, J., Geraedts, J. M. P., \& Wang, C. C. L. (2019). Color-Based Proprioception of Soft Actuators Interacting with Objects. IEEE/ASME Transactions on Mechatronics, 24(5), 1964-1973. [8766864]. https://doi.org/10.1109/TMECH.2019.2929818

\section{Important note}

To cite this publication, please use the final published version (if applicable).

Please check the document version above.

\section{Copyright}

Other than for strictly personal use, it is not permitted to download, forward or distribute the text or part of it, without the consent of the author(s) and/or copyright holder(s), unless the work is under an open content license such as Creative Commons.

Takedown policy

Please contact us and provide details if you believe this document breaches copyrights. We will remove access to the work immediately and investigate your claim. 


\title{
Color-Based Proprioception of Soft Actuators Interacting with Objects
}

\author{
Rob B.N. Scharff, Student Member, IEEE, Rens M. Doornbusch, Eugeni L. Doubrovski, Jun Wu, \\ Jo M.P. Geraedts Member, IEEE and Charlie C.L. Wang ${ }^{\dagger}$ Senior Member, IEEE
}

\begin{abstract}
Actuators using soft materials feature a large number of degrees of freedom. This tremendous flexibility allows a soft actuator to passively adapt its shape to the objects under interaction. In this paper we propose a novel proprioception method for soft actuators during real-time interaction with priorly unknown objects. Firstly, we design a color-based sensing structure that instantly translates the inflation of a bellow into changes in color, which are subsequently detected by a miniaturized color sensor. The color sensor is small and thus multiple of them can be integrated into soft pneumatic actuators to reflect local deformations. Secondly, we make use of a Feedforward Neural Network (FNN) to reconstruct a multivariate global shape deformation from local color signals. Our results demonstrate that deformations of the actuator during interaction, including sigmoid-like shapes, can be accurately reconstructed. The accurate shape sensing represents a significant step towards closed-loop control of soft robots in unstructured environments.
\end{abstract}

Index Terms-Shape Prediction, Color Sensor, Pneumatic Actuator, Sensor Fusion, Soft Robotics

\section{INTRODUCTION}

D UE to their intrinsic compliance, actuators made from soft materials have shown their great potential in many tasks such as grasping. The flexibility of soft materials allows the actuator to passively adapt its shape in response to physical contacts with objects. The control of soft robot actuators is typically performed in open loop. For grasping objects with known geometry, the curvature of a pneumatic actuator can be controlled through the applied pressure and mechanical programming (e.g., [1], [2]). However, for precise operations in unstructured environments and dexterous manipulation [3], mechanical programming becomes ineffective and closedloop control is needed. A fundamental step towards feedback control of soft actuators is the development of proper proprioception methods [4], [5].

Our work focuses on soft pneumatic actuators. This type of actuators is commonly used in soft robotics. It uses pressurized air to inflate chambers. The asymmetry in the geometry of internal chambers or physical properties of constituent materials drives the shape to the intended deformation [6]. Specifically,

R.B.N. Scharff, R.M. Doornbusch, E.L. Doubrovski, J. Wu and J.M.P. Geraedts are with the Department of Design Engineering, Delft University of Technology (TU Delft), The Netherlands.

C.C.L. Wang is with the Department of Mechanical and Automation Engineering, The Chinese University of Hong Kong at present. Part of this work is conducted when he was working at TU Delft.

J.M.P. Geraedts and C.C.L. Wang are also associated with the TU Delft Robotics Institute.

${ }^{\dagger}$ Corresponding Author: cwang@mae.cuhk.edu.hk

Manuscript prepared on May 5, 2018. downward bending can be realized through pressurization of an air chamber with an extensible top layer and an inextensible bottom layer [7]. In this paper, we present a novel method to accurately sense and reconstruct the bended shape of soft pneumatic actuators during real-time interaction with objects.

\section{A. Related Work}

1) Sensing in soft actuators: The use of exteroceptive sensing devices such as visual tracking systems has been reported in soft robotics (e.g., [8], [9], [10]). However, proprioceptive sensing approaches have the potential to create more compact sensing systems and further improve the autonomy of soft robots. This requires sensors that can be embedded in soft actuators. Ideally such sensors shall be bendable and/or stretchable, and thus do not prevent the deformation of soft actuators. An overview of sensing principles for soft actuators can be found in [11], [12], [13].

A commonly used approach to realize position sensing in soft robots is through embedded channels filled with conductive inks [14], [15] or liquid metals [16], [17] that change electrical resistance upon deformation. Other resistive sensors make use of conductive polymers that can be directly 3D-printed [18], [19], or off-the-shelf flex sensors [20]. An overview of resistive flex sensors is given by Saggio et al. [21]. An alternative use of electrical properties includes highly flexible capacitive sensors built from conductive fabrics and silicone [22] or aluminum and silver layers on an elastomer surface [23].

Some contactless sensors have also been developed, using optics [24] or magnetics [25]. For instance, Zhao et al. developed stretchable optical waveguides in soft prosthetic hands to feel the shape and softness of objects [26], [27].

Many of existing sensors suffer from elastic hysteresis, fabrication complexity and compromise in compliance, or can hardly be extended for the integration of multiple sensors throughout the actuator, which is necessary for reconstructing complex global deformation. Our color-based sensing approach overcomes all these difficulties.

2) Actuator model: Besides the development of accurate sensors, the actuator model which describes the global deformation and to which the sensing is calibrated is important for feedback control. Existing algorithms for shape reconstruction of soft robots are oversimplified, and do not address complex / local deformations [12]. The global deformation of soft actuators is typically described by using a simple descriptor such as average curvature [26], [17], [25], [28] or bending angle [18], [19], [20]. These univariate models are representative 

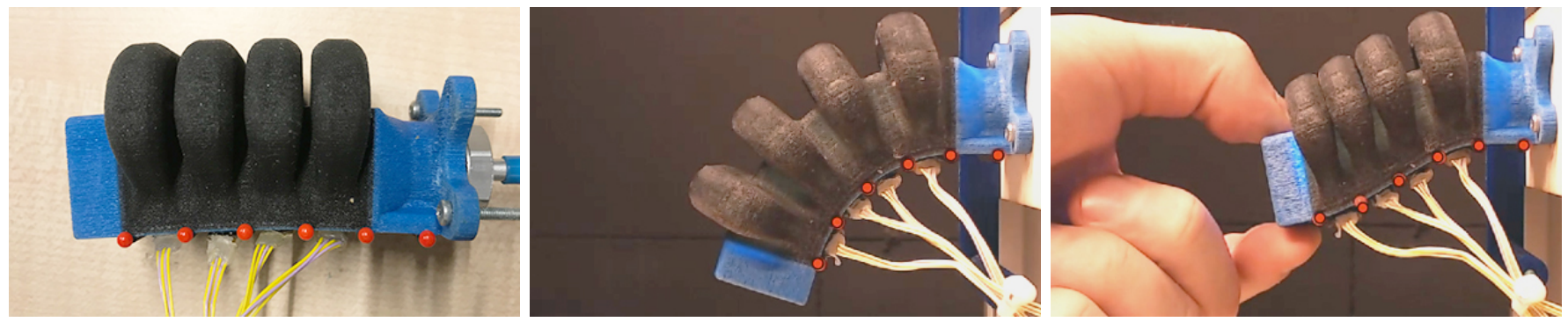

Fig. 1: A soft pneumatic actuator with red glass-headed pins inserted in the inextensible layer, serving as makers for the training of the neural network (left). The deformed shape is represented by the red dots, the locations of which are predicted from four color sensors embedded in the actuator (middle and right). Note that the predicted locations of the red dots accurately match the red pins, in the absence of object interaction (middle) and during interaction with objects (right).

for bending in the absence of object contacts. However, the soft actuator continuously adapts its shape during interaction with objects. A single parameter thus becomes ineffective to accurately describe the global deformation (e.g., the sigmoid curve in Fig. 11. Other soft robot sensing methods [27], [29] focus on object detection rather than shape sensing, bypassing the need for a continuous actuator model.

While the kinematics can be analytically derived for robots composed of rigid links, the deformation and motion for soft robots involves a lot more degrees of freedom (DoFs). A promising direction for soft robots is the use of learning algorithms to directly predict the deformed shape of the actuator based on sensor values. Giorelli et al. [30], [31] used a Feed-forward Neural Network (FNN) to learn the tip position of a cable-driven soft tentacle based on the cable forces. Runge et al. [32] suggested Finite Element Analysis (FEA) based training to learn a kinematic model of a soft pneumatic actuator through a neural network. Neural networks have also been applied to calibrate soft sensors to estimate the magnitude and the location of a contact pressure [33]. Wall et al. [5] used polynomial regression learning methods to find the most effective layout of sensors from a redundant layout of sensors on a soft pneumatic actuator. In parallel with our work, Van Meerbeek et al. [34] predict the bending and twisting of a soft foam with embedded optical fibers, comparing several learning techniques. Thuruthel et al. [35] show that temporal relations in the sensor data can be used to predict the fingertip position of a soft actuator using a recurrent neural network known as Long Short-Term Memory (LSTM). However, neither of them has demonstrated the capability of predicting the deformed shape of a soft gripperdeformation generated by unknown interactions as shown in our work. An FNN is used in this paper to reconstruct the global deformation. It demonstrates that learning algorithms can be used to fuse information of multiple sensors that overlap with each other. Benefited from our novel color-based sensing approach, this is the the first approach that can accurately predict the global shape of an actuator interacting with objects.

\section{B. Overview and Organization of the Work}

The basic idea of our shape sensing approach is to translate the bending that occurs in the extensible parts of soft actuators into a measurable change in colors. The color signals are captured by a miniaturized color sensor that is embedded in the inextensible layer of the soft actuator. To translate structural deformation into changes in color, we developed a multi-color structure that can be fabricated by $3 \mathrm{D}$ printing. With the local deformations of the actuator detected by a set of embedded color sensors, we make use of an FNN to reconstruct the global deformation. As shown in Fig. 1, the deformed shape of a pneumatic actuator can be accurately reconstructed by the proposed method, even after the inextensible bottom layer is largely distorted into a sigmoid-like curve. The reconstructed global deformation can provide much more information than (averaged) curvature or bending angle as used in many existing approaches (e.g., the 3D printed soft hand in [1]) to the control system of soft actuators.

Note that, this paper is an extension of our recent research [36] which proved the principle of color-based curvature sensing. Here, we extend it to reconstruct the multivariate shape deformation by integrating multiple color sensors. To this end, machine learning is employed for deformation reconstruction.

The rest of this paper is organized into five sections. Section III presents the developed color-sensing method and discusses the design and fabrication of the actuator with integrated signal generators. In order to generate training data set for accurately estimating a deformed shape, the method for data acquisition is introduced in Section III. Both the hardware setup and the steps of sampling will be discussed. After that, an FNN-based method is employed in Section IV to fuse the color signals captured by multiple sensors to reconstruct the deformed shape of an actuator. The experimental results will be presented in Section $\mathrm{V}$ and the paper ends with conclusion in Section VI

\section{COLOR-BASED SENSING}

\section{A. Sensing Principle}

The sensing principle is based on multi-color structures, the color and intensity of which gradually change upon deformation. The changes can be observed by cost-effective color sensors. To substantiate this principle, as illustrated in Fig. 2, inside a soft pneumatic actuator $\Omega$-shaped bellows are colored by two distinct colors, distinguishing different parts of a bellow. Beneath each bellow a color sensor, embedded 


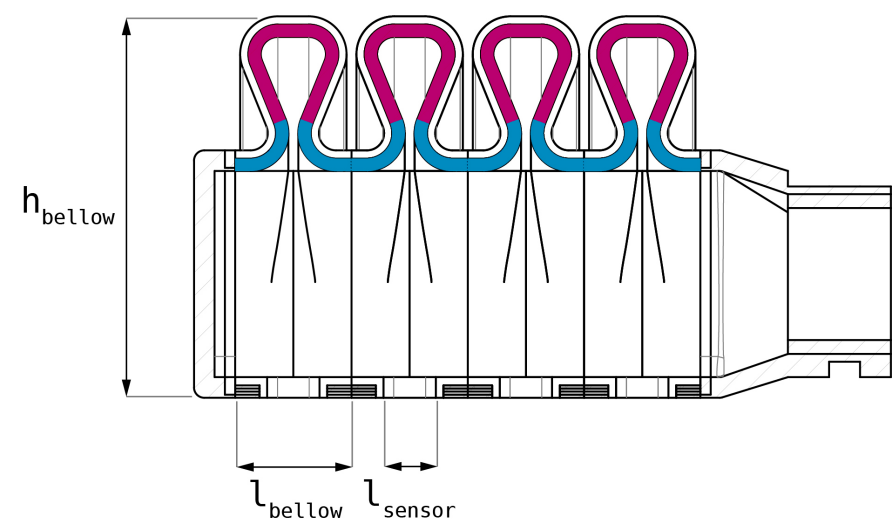

Fig. 2: Illustration of multi-color bellows inside a soft pneumatic actuator for translating deformation into changes in color. The dimensions of the bellow used in our experiments are $l_{\text {sensor }}=5.1 \mathrm{~mm}, l_{\text {bellow }}=11.3 \mathrm{~mm}$ and $h_{\text {bellow }}=$ $37.0 \mathrm{~mm}$.
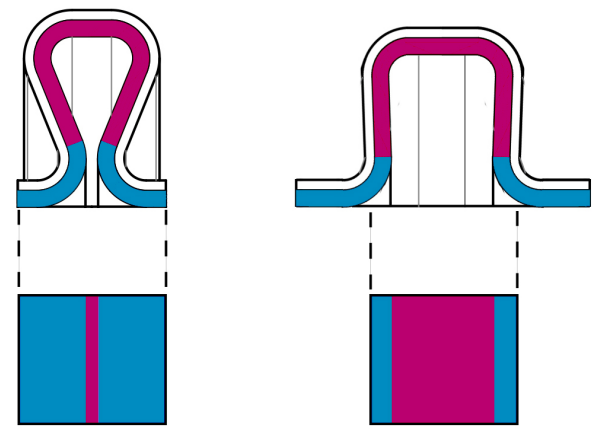

Fig. 3: Illustration of a bellow before and after being pressurized. The pattern of colors that can be captured through the sensing window is shown at the bottom.

in a plug, is plugged into the inextensible layer of the bending actuator. Upon pressurization of the air chamber, the bellows inflate, exposing a large area of the initially occluded color (i.e., magenta) to the window of the color sensor (see Fig. 3). Consequently, the measurement of the sensor changes accordingly. This sensing principle can be applied to bellowbased pneumatic actuators of different shapes and dimensions. The length of the inflatable part in our design is $45.2 \mathrm{~mm}$. The main dimensions of the actuator are indicated in Fig. 2. Although the measurements depend on the particular actuator design, a general learning procedure, which will be presented in Section IV can be conducted to map the measurements of the color sensors to the deformed shapes of an actuator.

\section{B. Sensor Crosstalk}

Although each color sensor is influenced most by the signal generated by the bellow directly above it, it is also influenced by adjacent bellows, as illustrated in Fig 4 . The degree of influence is not constant but depends on the deformation of the actuator.

This 'crosstalk' between sensors would normally be undesirable, as it complicates signal processing. We propose the

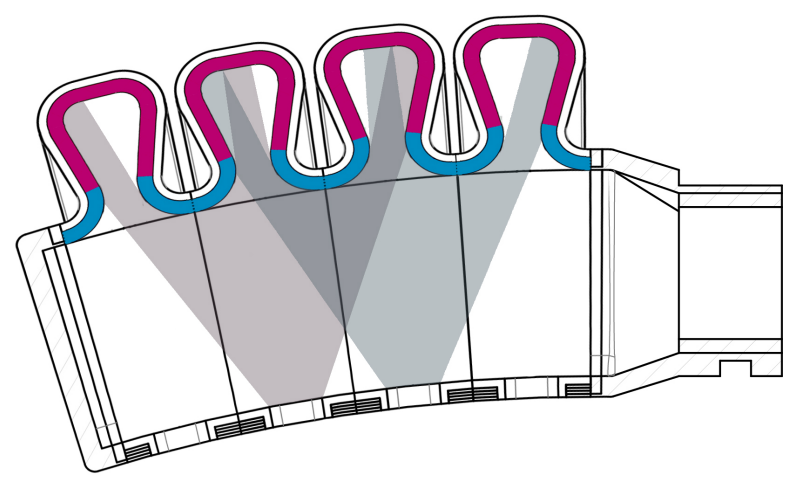

Fig. 4: Illustration of sensor crosstalk - each sensor captures signals generated by the bellow directly above it and the neighboring bellows.
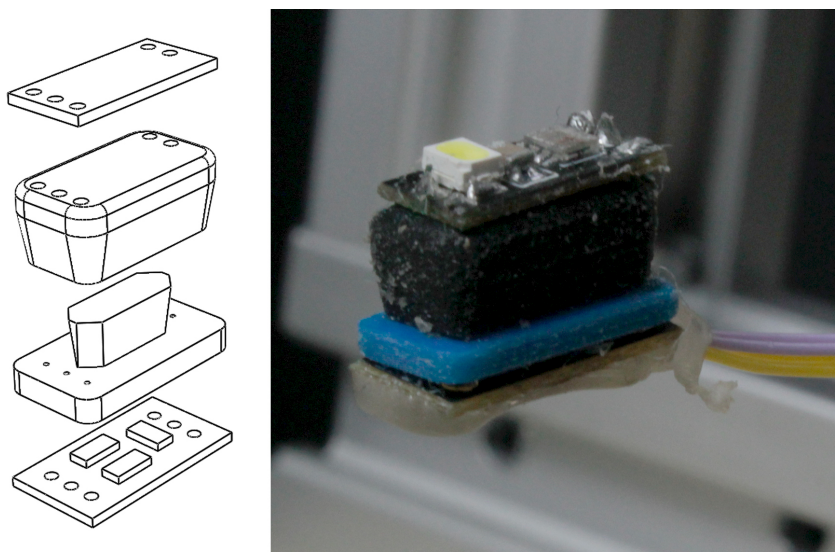

Fig. 5: Both an exploded view of the sensor plug design (left) and the assembled sensor plug (right) are shown. In the exploded view (left), from the bottom up - the lower PCB, rigid bottom, flexible plug and upper $\mathrm{PCB}$. Note that the rigid bottom and flexible plug are printed as one piece.

use of neural network based method for fusion, which does not suffer from this undesirable effect as it can learn the deformed shape of an actuator at a global level. In fact, as will be demonstrated in Section $\mathrm{V}$ this crosstalk situation can actually be used to reduce the number of sensors. This would not be possible when using a separate sensor calibration for each bellow segment.

\section{Color Sensors}

A color sensor is plugged into the inextensible layer of the actuator at each bellow segment. To reduce the size of color sensors, we customized the color sensor with a design of twolayer PCBs, mounted on a 3D-printed plug (see Fig. 5). The shape of the plug creates an airtight seal. The detailed design of the sensor can be found in Appendix A. The color sensor has a built-in LED that emits light to the multi-color structures above it. Similar to the sensor crosstalk, the light is also shed on the neighboring bellow segments.

For color sensing, the TCS34725 light-to-digital converter is used. The sensor returns four values, namely Red, Green, 


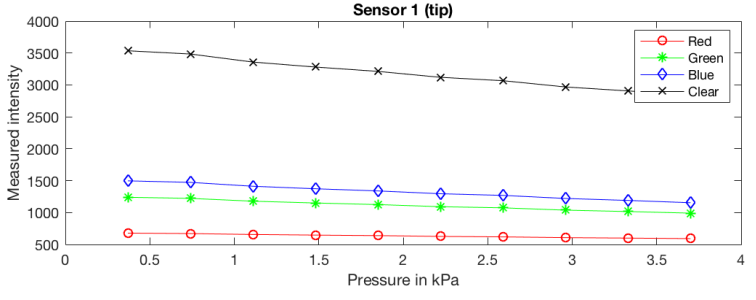

(a) Raw RGBC data

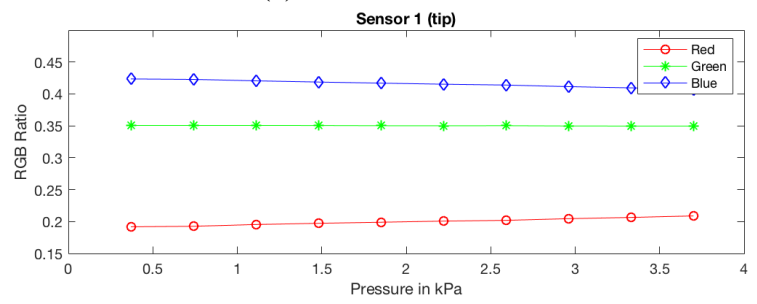

(b) RGB normalized by the Clear value

Fig. 6: (a) Raw RGBC data of a color sensor measured at pressure intervals during unobstructed bending. Besides a clear decrease in intensity due to an increased measuring distance, a change in color can be observed as well. (b) RGB normalized by the Clear value. The ratio of red gradually increases from 0.1918 to 0.2088 , while the blue ratio decreases from 0.4235 to 0.4071 .

Blue and Clear (RGBC), for each exposure. The clear value is IR filtered light, which gives an indication of overall light intensity. The sensor integration time is set to $50 \mathrm{~ms}$ and the gain to $60 X$. An Adafruit Feather HUZZAH with ESP8266 reads the sensor-data of the four sensors with a negligible delay between each sensor by using a TCA9548A I2C Multiplexer. The Feather HUZZAH runs code using adafruit's TCS34725 library. A command to collect a data sample is sent from the main Matlab script through serial communication. The collected data is then sent back to Matlab.

\section{Materials \& Fabrication}

The embodiment of our design is fabricated on a Stratasys Objet 350 Connex3 multi-material 3D printer, which uses Material Jetting technology and can print combinations of up to three different building materials in addition to a water soluble support material. The overall structure of the actuator is printed with the flexible Agilus 30 Black, while the multicolored bellows are printed with the rigid VeroCyan and the rigid VeroMagenta. Note that pure blue or red colors are not available for this 3D printing system. As the colored materials are rigid, we want to apply a layer as thin as possible. VeroMagenta and VeroCyan but not VeroYellow materials are chosen as VeroYellow has a higher translucency.

The Agilus 30 Black has an elongation of $220 \sim 270 \%$ at break and a tensile strength of $2.4 \sim 3.1 \mathrm{MPa}-$ as documented by Stratasys. The $R G B$ values of the VeroMagenta (VM) and VeroCyan $(\mathrm{VC})$ materials are $R G B_{\mathrm{VM}}=(166,33,98)$ and $R G B_{\mathrm{VC}}=(0,93,127)$ respectively.

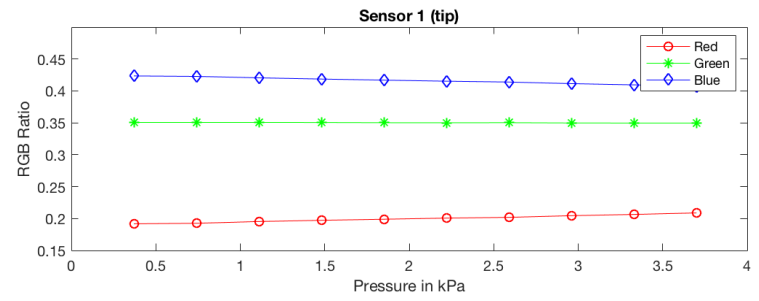

(a) RGB ratio at the first measurement: [0.1918, 0.3508, 0.4235]. RGB ratio at the last measurement: [0.2088, 0.3496, 0.4071]

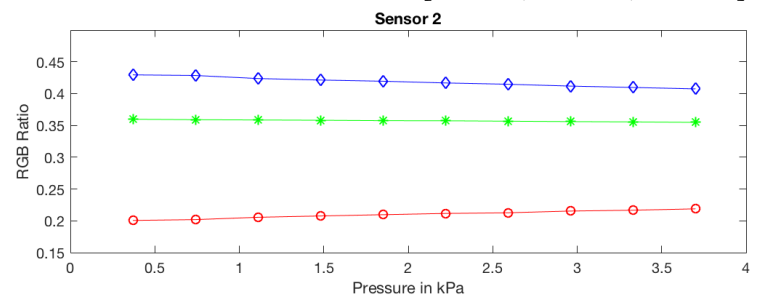

(b) RGB ratio at the first measurement: [0.2006, 0.3597, 0.4295]. RGB ratio at the last measurement: [0.2188, 0.3548, 0.4074]

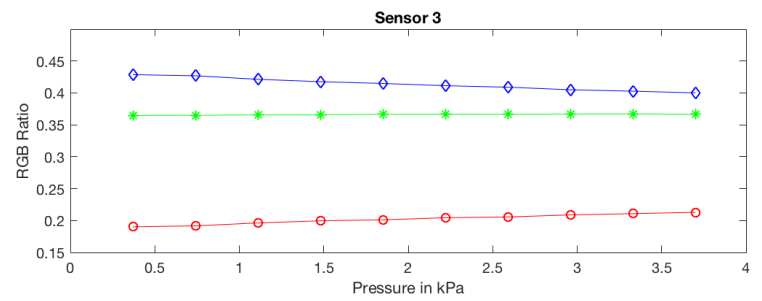

(c) RGB ratio at the first measurement: [0.1902, 0.3646, 0.4286]. RGB ratio at the last measurement: [0.2129, 0, 3666, 0.4001]

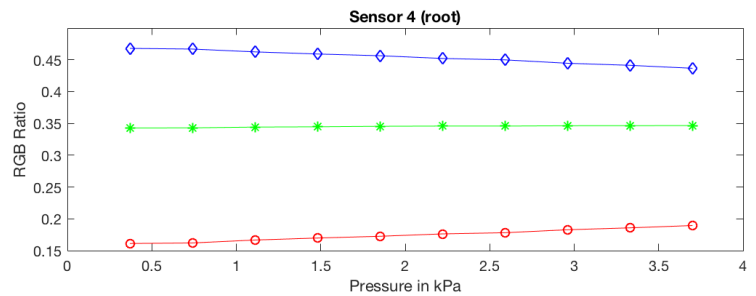

(d) RGB ratio at the first measurement: [0.1612, 0.3428, 0.4679]. RGB ratio at the last measurement: [0.1894, 0.3466, 0.4367]

Fig. 7: RGB values of the four sensors plugged underneath the four bellows. The RGB ratio data is obtained by dividing the raw RGB values by the Clear value. For each sensor there is a clear increase in red and decrease in blue.

\section{E. Color Signal}

Figure 6,a) shows the raw data that was obtained by reading out the RGBC-data of a color sensor during unobstructed progressive bending. A clear decrease in overall intensity can be observed. This is due to the increased measurement distance towards the hidden (magenta) elements that appear upon bending, as well as the decrease in overlap of the light of the LEDs upon bending (similar to the crosstalk in the sensors explained in subsection [I-B]. Besides the change in intensity, a color change can be observed as well. As shown in Fig. 6(b) the raw RGB values are normalized by dividing the raw RGB values by the Clear value. The normalized RGB color codes show a clear increase in red and a clear decrease in blue, confirming the appearance of a larger magenta area. 


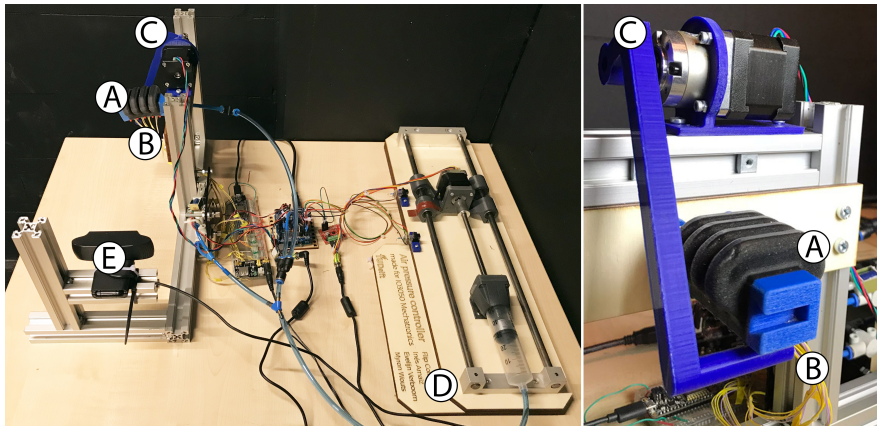

Fig. 8: The data acquisition setup: (A) the soft pneumatic actuator, (B) the four color sensors, (C) the variable obstacle, (D) the pressure regulator and (E) the camera.

Similar observations can be made from sensors embedded in other slots, as can been seen in Fig. 7. We do not observe a clear trend in the green ratio upon bending. This can probably be attributed to the fact that the VeroCyan material contains less green than real cyan while the VeroMagenta contains more green than real magenta, and the AgilusBlack also contains some green.

\section{DATA ACQUisition}

This section focuses on describing how the data, used in the learning for reconstruction procedure, was acquired. We built a setup that randomly changes the actuation pressure and the position of an obstacle. It then captures an image of the deformed actuator and collects the measurements of the color sensors. Two readings are made during one loop - due to hysteresis the actuator will slowly deform after reaching a certain pressure. Both readings are collected into the set of training samples. This allows for generating more samples in a shorter timespan. The sample data collection process is automatic. The main loop, running in Matlab, looks as follows:

1) Set random obstacle angle;

2) Set random actuation pressure;

3) Take first picture;

4) Read out data of the color sensors;

5) Wait for 2 seconds;

6) Take second picture;

7) Read out data of the color sensors;

8) Set pressure back to zero;

9) Set obstacle back to default position.

Our data acquisition setup is illustrated in Fig. 8. Its main components are discussed in the following.

We took samples from 500 random pressure/obstacleconfigurations for the training. Two samples were taken for each configuration, so a total of 1000 data samples were collected. The total data collection procedure took less than 5 hours.

\section{A. Shape of Actuator}

We used the soft actuator design as discussed in Section $\Pi$ II Our actuator has four bellows. Red glass-headed pins are inserted into designated pin holders in the inextensible layer, serving as markers (see Fig. 1). An additional marker is placed on the origin of the actuator. The positions of the origin marker and the first marker are fixed (see Fig 9), and the distance between the markers is known. These markers are used to determine the orientation of the actuator and to convert from pixel positions to metric positions in the unit of $\mathrm{mm}$. As a result, the shape of an actuator can be obtained by processing the images captured by camera.

\section{B. Variable Obstacle}

An obstacle is attached through a 3D-printed arm linked to to a stepper motor (see Fig $8(\mathrm{C})$ ), and thus its location can be varied by the stepper. To (roughly) align the trajectories of the obstacle and the actuator and thus create valid obstructing, the arm has a length of $140 \mathrm{~mm}$ and its axis is located $100 \mathrm{~mm}$ above the inextensible layer of the actuator. At the start of one loop, the obstacle angle is randomly chosen between 15 and 32 degrees. We have a resolution of 65 uniform steps within this range. We use an Arduino Uno and Pololu DRV8825 stepper driver to control the stepper (see Fig 8 C)). The random obstacle position is controlled the main Matlab program through serial communication.

\section{Pressure Regulator}

The actuator requires low pressures to generate relatively large changes in actuator deformation. For the data acquisition, we need to generate stable actuator deformations with a high resolution within the actuator's actuation range of 0 to $5 \mathrm{kPa}$. This is realized by a syringe based pressure regulator. Specifically, a stepper motor moves the plunger of a syringe to add air to, or subtract air from, the actuator (see the illustration in Fig $8(\mathrm{D}))$. The pressure inside the actuator is measured using an NXP MP3V5050GC6U pressure sensor with a range of $0-50 \mathrm{kPa}$. A desired pressure can then be achieved by a PID-controller using Arduino.

\section{Camera}

We use a Logitech C922 Pro Stream Webcam to capture the positions of markers. The camera is placed at a distance of $250 \mathrm{~mm}$ to the actuator markers. We capture images with a resolution of $1920 \times 1080 \mathrm{p}$. The camera settings are set manually and are kept constant over all captured images. A sample of the raw image captured by camera is shown in Fig. 9

\section{E. Sampling Strategy}

During a pilot acquisition, the pressure was varied between 0 and $5 \mathrm{kPa}$ and the obstacle angle between 0 and 32 degrees, as shown in Fig. 10. However, our experiments suggest that a relatively high amount of constrained configurations in the training data can improve the training results. Therefore, we increased the minimum value of the obstacle angle to 14.5 degrees. The results of the final sampling are shown in Fig. 11] Note that the maximum pressure was also reduced to increase the life-span of the actuator. 


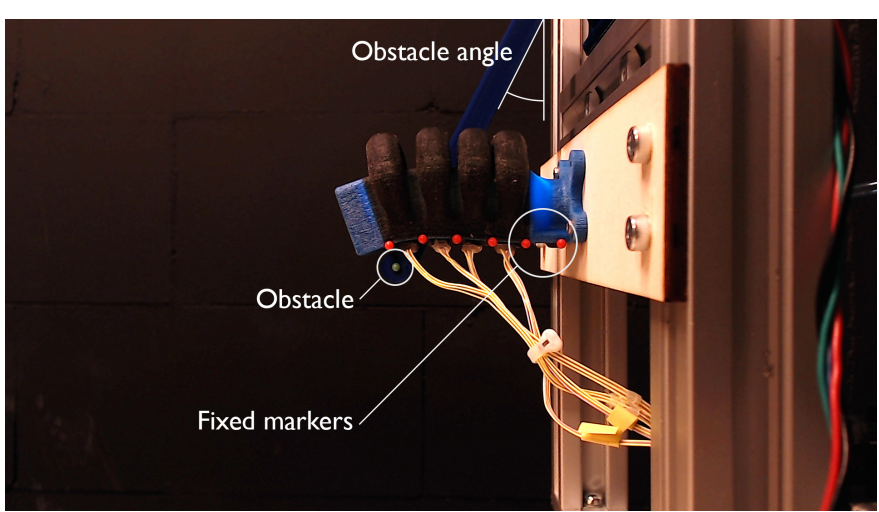

Fig. 9: An example sample of the raw camera data when the actuator interacts with an obstacle. Six red markers are used to describe the actuator's shape. The two most right markers at the root of actuator are fixed.

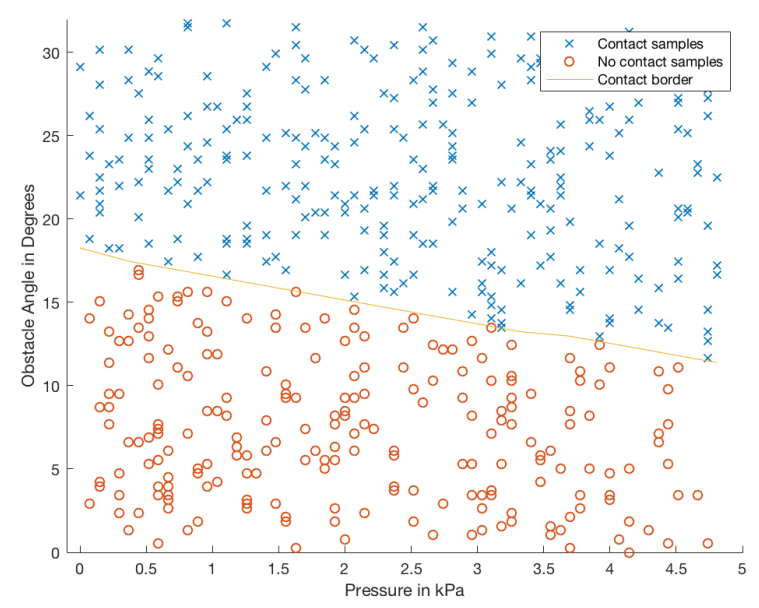

Fig. 10: Samples that were collected during a pilot acquisition and training, where the samples indicated by a cross are interacting with the obstacle. Although the training performed quite well on the test-data and we can well predict the unobstructed bending, the learned shape predictor had inferior results when confronted with other types of obstacles not presented in the training set.

\section{DEFORMATION RECONSTRUCTION}

The global deformation of an actuator can be represented by the coordinates of the six markers in the inextensible layer. This section discusses the reconstruction of the deformation from the sensor data. We use a Feed-forward Neural Network to train the global actuator shape based on the sensor data. To train the network, we use the RGBC-data of the sensors as inputs and the coordinates of six markers as outputs. The RGBC-data of the four sensors can be directly fed into the FNN. Some processing is needed for the marker coordinates as the set of training data.

First, we undistort the captured images using the parameters obtained from a multi-plane camera calibration. Our calibration has a Mean Pixel Error of 0.44. Using the fixed distance of $11.4 \mathrm{~mm}$ between the first two markers on the right, we make

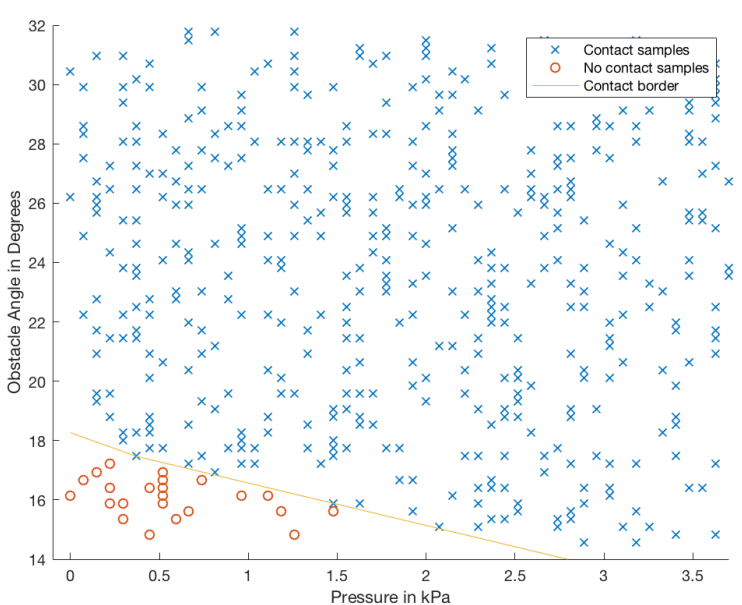

Fig. 11: The final sampling strategy that was applied to collect the training data. After introducing more contacted samples into the training set, the predictor can reconstruct very accurate shape of an obstructed actuator. the predictor gives a better reconstruction of obstructed actuator shapes, while maintaining its accuracy on unobstructed actuator shapes.

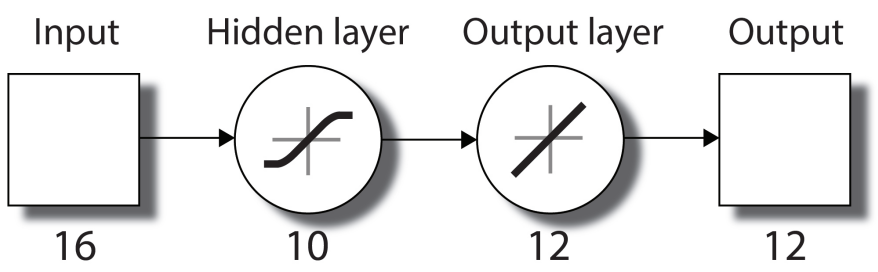

Fig. 12: Graphical representation of the Neural Network configuration used in our training process and the shape predictor.

a conversion from pixel-scale to millimeter-scale at the rate of $6.8875: 1$. This means the mean error of the calibration is $0.064 \mathrm{~mm}$. We remove the red cables shown on the right of Fig. 9. by simply cropping out the right of the picture to a resolution of $1300 \times 1080$ p. After cropping, we use Matlab's Computer Vision System Toolbox functions to extract the $\mathrm{x}$ and y-coordinates of the six red markers in millimeter. The positions of the markers are ordered by solving a Travelling salesman problem. These values are used as outputs of the FNN.

A graphical representation of the FNN is shown in Fig. 12 The two-layer network has a hidden layer with ten neurons. The hidden layer uses a sigmoid transfer function and the output layer uses a linear transfer function. We have a total of 16 inputs (four sensors with four measurements) and 12 outputs ( $\mathrm{x}$ - and $\mathrm{y}$-coordinates of six markers).

Unless otherwise specified, we use a block division of our samples, where the first $70 \%$ of our samples are used for training, the next $15 \%$ as validation set and the remaining $15 \%$ as test set. We apply the Levenberg-Marquardt algorithm to train the network, and obtain a shape predictor with the raw data of the sensors as input. 


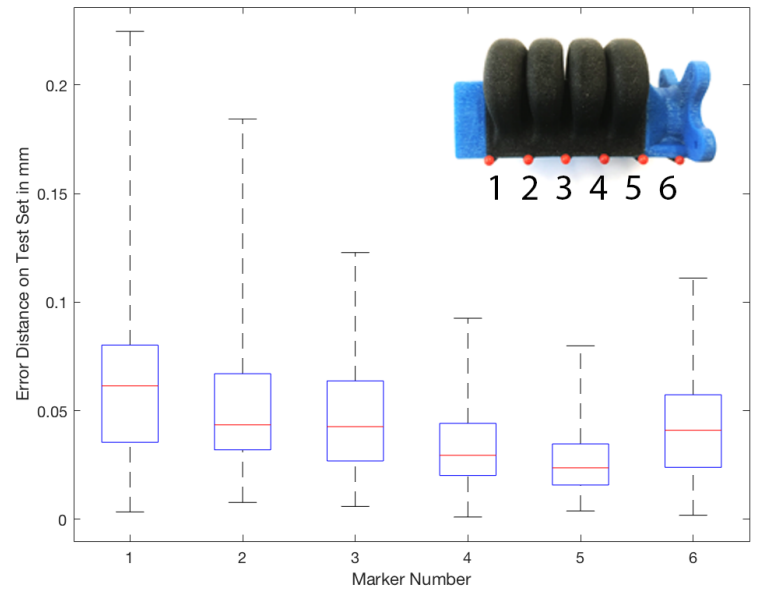

Fig. 13: The error distance between the real and the predicted positions for each marker. The box plot shows the median, the 25th / 75th percentile, and the minimal / maximal bounds.

\section{RESULTS}

\section{A. Accuracy}

The error distance between the real and the predicted marker positions is calculated for all marker positions of the samples in the test-set. The error distance is calculated as

$$
\epsilon_{i}=\sqrt{\left(x_{\mathrm{R}_{i}}-x_{\mathrm{P}_{i}}\right)^{2}+\left(y_{\mathrm{R}_{i}}-y_{\mathrm{P}_{i}}\right)^{2}},
$$

where subscripts $\mathrm{R}$ and $\mathrm{P}$ indicate the real and predicted coordinates respectively and $i$ indicates the sample number. The results are given in Fig. 13. It shows that the median prediction error is lower than $0.1 \mathrm{~mm}$ for all marker coordinates. As would be expected, the largest error is observed on the marker at the tip of the actuator, and the error decreases when moving closer towards the root of the actuator. The error is smallest for the marker at location ' 5 '.

To validate the advantage of using multiple sensors, we trained the FNN for all possible sensor quantities and sensor configurations. In Fig. 14, the mean Mean-Squared Error (MSE) of each configuration is plotted in a bar graph. We trained each configuration 10 times in these experiments.

The results show that the sensor at the root of the actuator (i.e., the configuration ' $\mathrm{COOO1}$ ') individually provides the most complete information (with the mean MSE: $8.542 \times 10^{-3} \mathrm{~mm}$ ). As an individual sensor, configuration 'C0010' (with the mean MSE: $6.551 \times 10^{-2} \mathrm{~mm}$ ) provides better information than the configuration where the sensor is placed at the second bellow from the tip (i.e., ' $\mathrm{CO100}$ ' with the mean MSE of $2.749 \times 10^{-1} \mathrm{~mm}$ ). However in a two sensor configuration, a combination of the second and the fourth sensors from the tip is the best (i.e., 'C0101' with the mean MSE: $\left.1.818 \times 10^{-3} \mathrm{~mm}\right)$ ) as the second sensor contains information that the root sensor is missing. The results show that two well-placed sensors can already achieve very accurate results and only small improvements are realized by adding more. This demonstrates that each sensor indeed captures information from neighboring bellow segments as well. Note

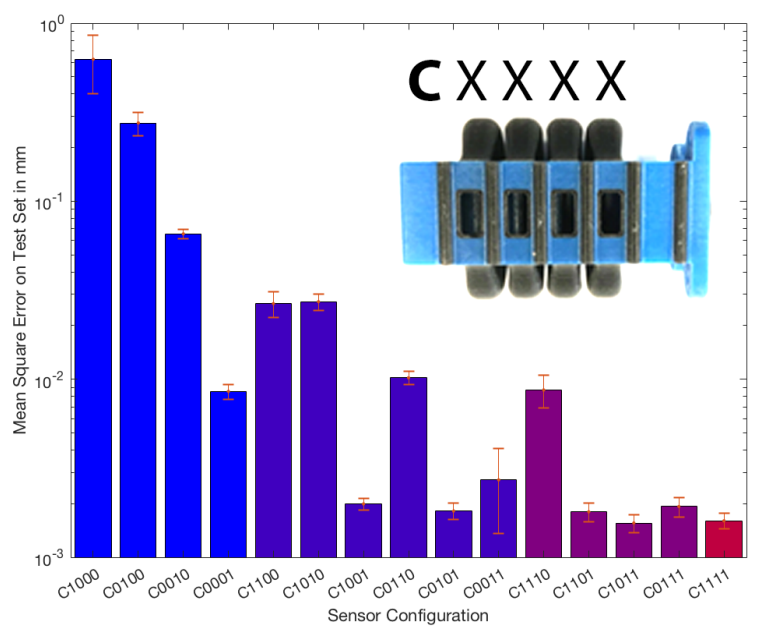

Fig. 14: The mean MSE of the predicted outputs on the test-set for all 15 possible sensor configurations. Each configuration was trained 10 times. The standard deviation is indicated on the error chart.

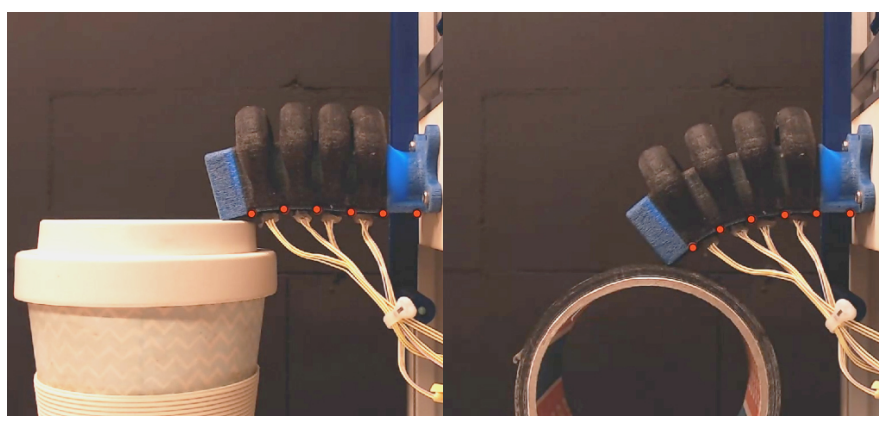

Fig. 15: While projecting the predicted positions of markers onto the camera images, it can find that our method also gives very accurate prediction in scenarios that are different from the training set. Some have complicated curvatures - e.g., the ' $S$ '-shape shown in the left figure.

that we train the predictor for the separate sensors by selecting the sensor outputs of the sensor of interest from the same data acquisition, in which the LEDs of all sensor plugs are on.

Our training based predictor also performs well on objects different from the trained obstacles. This is demonstrated in Fig. 15, where we test the sensing method on other objects.

The position of the actuator can be predicted in real time. In the supplementary video (https://youtu.be/zgOexw8YLQc), the predicted coordinates are projected on top of the live camera images to demonstrate the accuracy of the method in various scenarios. Due to the simplicity of the FNN configuration, the capturing (50 ms for the integration time) and evaluation $(0.232 \mathrm{~ms})$ of a new set of inputs is very efficient. This makes our approach very suitable for real-time feedback control. The relative slow loop in the supplementary video is due to the visualization of the $1920 \times 1080 p$ camera image 


\section{B. Robustness}

We tested the actuators under some extreme conditions to explore how it would perform on load cases that were not included in the training set. Although some accuracy is lost, the neural network is still able to predict most of the extreme load cases quite well. The right of Fig 1 shows the prediction on a sigmoid-curved actuator shape that is realized by bending and rotating the actuator's tip at the same time.

It should be noted that our light-based sensing method is almost not affected by external lighting conditions. This is due to the light absorbing black flexible air chamber and the bright LEDs placed in the sensor plugs. We demonstrate this in the supplementary video where the actuator is tested in dark and extremely bright conditions.

Another aspect of robustness, repeatability, has also been tested in our experiments. As demonstrated in the supplementary video, sensors can be pulled out and inserted back in while regaining nearly the same result.

\section{Importance of Color Signal}

The raw RGBC data in Fig. 6 showed that, due to intensity changes that occur upon bending, the Clear signal on itself was already a good indicator of the bending deformation, To test the performanceofwhen only using the Clear signal to predict an actuator's shape, we trained our network with the Clear signal of the sensors as inputs. The training was able to realize a mean MSE of $2.633 \times 10^{-3} \mathrm{~mm}$ on the test set (over 10 trainings) as compared to a mean MSE of $1.602 \times 10^{-3} \mathrm{~mm}$ when including the RGB data as addition. This proves that the changes in color upon bending are very necessary for improving the accuracy of shape prediction.

Whereas the mean MSE of the '1001' sensor configuration was very close to the ' 1111 ' configuration when using all RGBC-data as inputs $\left(1.994 \times 10^{-3} \mathrm{~mm}\right.$ and $1.602 \times 10^{-3} \mathrm{~mm}$ respectively), the mean MSE increases more significantly when only the Clear value is used $\left(5.165 \times 10^{-3} \mathrm{~mm}\right.$ for configuration '1001'). This means that the color signals are very important to ensure the robustness of our sensing approach.

When an application can accept the aforementioned error of $2.633 \times 10^{-3} \mathrm{~mm}$, fast and low-cost shape sensing can be achieved by embedding off-the-shelf photo-detectors such as LDRs, and LEDs into a soft pneumatic actuator fabricated in a single color. This is considered as a simplified version of our approach with reduced accuracy and robustness, which can be applied to more durable actuators fabricated by silicone casting.

\section{Comparison to Multivariate Polynomial Regression}

To demonstrate the effectiveness of a neural network, we compare the MSE of the training result to the MSE obtained when using Multivariate Polynomial Regression (MVPR). For both the MVPR and the FNN, we use the first 850 samples for training, and evaluate the accuracy of prediction (by MSE) on the remaining 150 samples. A multivariate linear regression (MVLR) results in a predictor with an MSE of $0.0377 \mathrm{~mm}$,

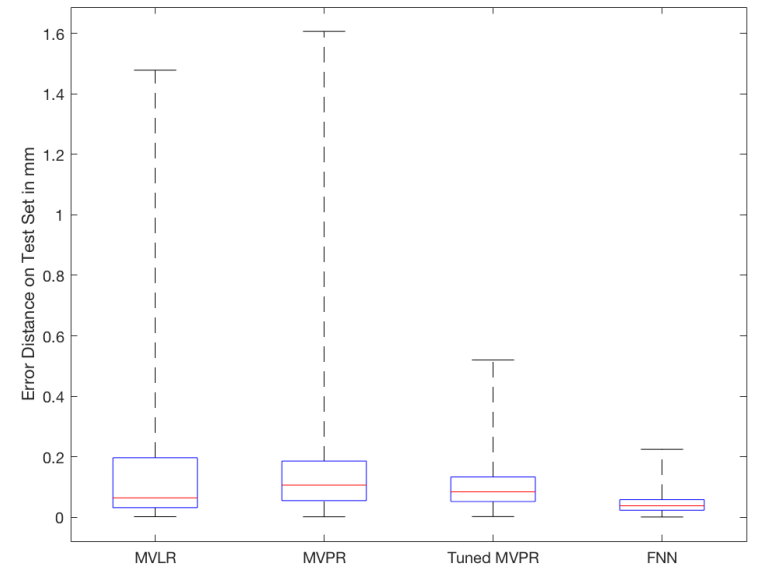

Fig. 16: The distances between the real positions and the positions predicted by the multivariate linear regression (MVLR), the quadratic multivariate polynomial regression (MVPR), the tuned MVPR and the FNN. The predictions generated by the FNN have smaller errors.

whereas a quadratic MVPR results in an MSE of $0.0788 \mathrm{~mm}$. Such errors are $23.5 \times$ and $49.2 \times$ that of the prediction by the FNN which has an MSE of $1.602 \times 10^{-3} \mathrm{~mm}$. After manually tuning the polynomials of the MVPR by removing some of the quadratic terms, we were able to obtain a prediction with an MSE of $0.0079 \mathrm{~mm}$, which is still $4.9 \times$ that of the corresponding MSE of the FNN. Moreover, the FNN-based predictor is robust and does not involve any manual tuning of parameters. Figure 16 shows the error distances over all markers by using the MVLR, the quadratic MVPR, the tuned MVPR and the FNN.

\section{CONCLUSION}

We have demonstrated a complete method to fabricate a soft pneumatic actuator with integrated color signal generators to realize accurate shape estimation in both obstructed and unobstructed situations. We have shown that our color-based sensing approach is robust and performs well on obstacles that were not included in the training data. This realization of accurate shape sensing is a significant step towards the application of soft robots in many dexterous tasks.

Our future work will focus on improving the performance on cases under specific loading conditions by collecting additional training data for these loadcases. Moreover, 3dimensional load cases and data collection could be used to include the effects of twisting of the bellow upon contact. Optimization could be performed to minimize the amount of samples needed without losing accuracy. Another existing problem is the long-term reliability of a 3D-printed actuator. It is worthy to work towards digital fabrication of soft actuators using materials with longer durability. Lastly, we will also work on how to estimate the complete actuator shape based on the predicted coordinates on the inextensible layer (e.g. by using elasticity simulation). 



Fig. 17: The design of the upper PCB (left with dimension: $10.80 \mathrm{~mm} \times 5.08 \mathrm{~mm}$ ) and the design of the lower PCB (right with dimension: $12.07 \mathrm{~mm} \times 6.35 \mathrm{~mm}$ ).

\section{ACKNOWLEDGEMENT}

Authors of this paper would like to thank financial support provided by the IDE faculty, Delft University of Technology as the research grant of CCL Wang to support the PhD study of R.B.N. Scharff.

\section{APPENDIX A}

\section{COLOR SENSOR PCB DESIGN}

This appendix discusses the sensor design. The design of the sensor plug has been shown in Fig. 5. The body of the plug is fabricated by a PolyJet 3D printing system using the rigid VeroCyan and the flexible Agilus30 photopolymers. Two Printed Circuit Boards (PCBs) - both customized - are mounted on the top and the bottom parts of the plug. The designs of these PCBs are shown in Fig. 17. The schemes of the upper and the lower PCBs can be found in Fig. 18 and Fig. 19 respectively. Wires are guided through the 3D-printed plug to connect the upper and lower PCB in an airtight way.

\section{ACKNOWLEDGMENT}

The authors would like to thank the research grant provided by the faculty of IDE at TU Delft. R.B.N. Scharff's research is fully supported by this grant.

\section{REFERENCES}

[1] R. B. N. Scharff, E. L. Doubrovski, W. A. Poelman, P. P. Jonker, C. C. L. Wang, and J. M. P. Geraedts, "Towards behavior design of a 3d-printed soft robotic hand," in Soft Robotics: Trends, Applications and Challenges. Springer International Publishing, 2017, pp. 23-29.

[2] K. C. Galloway, P. Polygerinos, C. J. Walsh, and R. J. Wood, "Mechanically programmable bend radius for fiber-reinforced soft actuators," in 2013 16th International Conference on Advanced Robotics (ICAR), 2013, pp. 1-6.
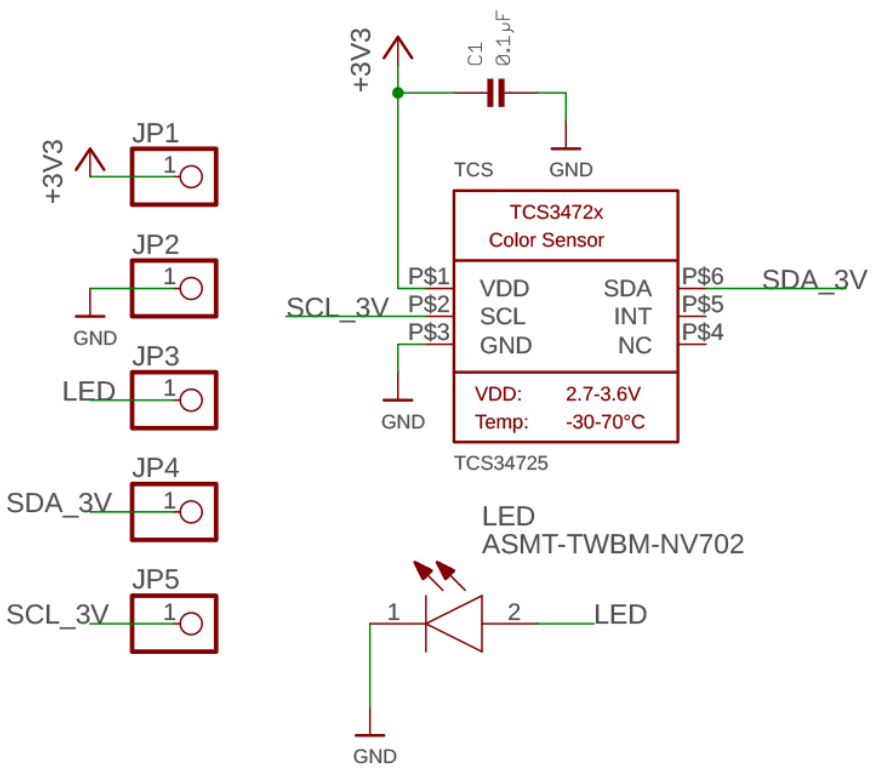

Fig. 18: Scheme of the upper PCB
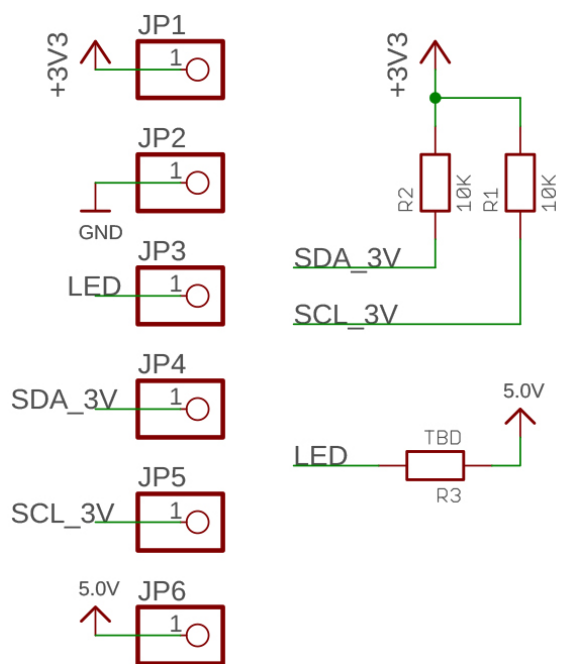

Fig. 19: Scheme of the lower PCB

[3] A. Gupta, C. Eppner, S. Levine, and P. Abbeel, "Learning dexterous manipulation for a soft robotic hand from human demonstrations," in 2016 IEEE/RSJ International Conference on Intelligent Robots and Systems (IROS), 2016, pp. 3786-3793.

[4] G. Gerboni, A. Diodato, G. Ciuti, M. Cianchetti, and A. Menciassi, "Feedback control of soft robot actuators via commercial flex bend sensors," IEEE/ASME Transactions on Mechatronics, vol. 22, no. 4, pp. 1881-1888, 2017.

[5] V. Wall, G. Zller, and O. Brock, "A method for sensorizing soft actuators and its application to the rbo hand 2," in 2017 IEEE International Conference on Robotics and Automation (ICRA), 2017, pp. 4965-4970.

[6] D. Rus and M. T. Tolley, "Design, fabrication and control of soft robots," Nature, vol. 521, pp. 467 EP -, 2015.

[7] B. Mosadegh, P. Polygerinos, C. Keplinger, S. Wennstedt, R. F. Shepherd, U. Gupta, J. Shim, K. Bertoldi, C. J. Walsh, and G. M. Whitesides, "Pneumatic networks for soft robotics that actuate rapidly," Advanced Functional Materials, vol. 24, no. 15, pp. 2163-2170, 2014.

[8] R. Deimel and O. Brock, "A novel type of compliant and underactuated robotic hand for dexterous grasping," The International Journal of Robotics Research, vol. 35, no. 1-3, pp. 161-185, 2016.

[9] A. D. Marchese, K. Komorowski, C. D. Onal, and D. Rus, "Design and control of a soft and continuously deformable $2 \mathrm{~d}$ robotic manipulation 
system," in 2014 IEEE International Conference on Robotics and Automation (ICRA), 2014, pp. 2189-2196.

[10] M. Luo, W. Tao, F. Chen, T. K. Khuu, S. Ozel, and C. D. Onal, "Design improvements and dynamic characterization on fluidic elastomer actuators for a soft robotic snake," in 2014 IEEE International Conference on Technologies for Practical Robot Applications (TePRA), 2014, pp. 1-6.

[11] S. Li, H. Zhao, and R. F. Shepherd, "Flexible and stretchable sensors for fluidic elastomer actuated soft robots," MRS Bulletin, vol. 42, no. 2, pp. $138-142,2017$.

[12] H. Wang, M. Totaro, and L. Beccai, "Toward perceptive soft robots: Progress and challenges," Advanced Science, p. 1800541, 2018.

[13] J. Hughes, U. Culha, F. Giardina, F. Guenther, A. Rosendo, and F. Iida, "Soft manipulators and grippers: A review," Frontiers in Robotics and AI, vol. 3, p. 69, 2016

[14] J. T. Muth, D. M. Vogt, R. L. Truby, Y. Mengüç, D. B. Kolesky, R. J. Wood, and J. A. Lewis, "Embedded 3d printing of strain sensors within highly stretchable elastomers," Advanced Materials, vol. 26, no. 36, pp. 6307-6312, 2014.

[15] J. C. Yeo, H. K. Yap, W. Xi, Z. Wang, C.-H. Yeow, and C. T. Lim, "Flexible and stretchable strain sensing actuator for wearable soft robotic applications," Advanced Materials Technologies, vol. 1, no. 3, p. 1600018,2016

[16] C. Majidi, R. Kramer, and R. J. Wood, "A non-differential elastomer curvature sensor for softer-than-skin electronics," Smart Materials and Structures, vol. 20, no. 10, p. 105017, 2011.

[17] J. Morrow, H. Shin, C. Phillips-Grafflin, S. Jang, J. Torrey, R. Larkins, S. Dang, Y. Park, and D. Berenson, "Improving soft pneumatic actuator fingers through integration of soft sensors, position and force control, and rigid fingernails," in 2016 IEEE International Conference on Robotics and Automation (ICRA), 2016, pp. 5024-5031.

[18] K. Elgeneidy, G. Neumann, M. Jackson, and N. Lohse, "Directly printable flexible strain sensors for bending and contact feedback of soft actuators," Frontiers in Robotics and AI, vol. 5, p. 2, 2018.

[19] Y. Yang and Y. Chen, "Innovative design of embedded pressure and position sensors for soft actuators," IEEE Robotics and Automation Letters, vol. 3, no. 2, pp. 656-663, 2018.

[20] K. Elgeneidy, N. Lohse, and M. Jackson, "Bending angle prediction and control of soft pneumatic actuators with embedded flex sensors - a data-driven approach," Mechatronics, vol. 50, pp. 234 - 247, 2018

[21] G. Saggio, F. Riillo, L. Sbernini, and L. R. Quitadamo, "Resistive flex sensors: a survey," Smart Materials and Structures, vol. 25, no. 1, p. 013001, 2016

[22] C. Lucarotti, M. Totaro, A. Sadeghi, B. Mazzolai, and L. Beccai, "Revealing bending and force in a soft body through a plant root inspired approach," Scientific Reports, vol. 5, pp. 8788 EP -, 2015.

[23] O. Atalay, A. Atalay, J. Gafford, H. Wang, R. Wood, and C. Walsh, "A highly stretchable capacitive-based strain sensor based on metal deposition and laser rastering," Advanced Materials Technologies, vol. 2, no. 9 , p. $1700081,2017$.

[24] M. K. Dobrzynski, R. Pericet-Camara, and D. Floreano, "Contactless deflection sensor for soft robots," in 2011 IEEE/RSJ International Conference on Intelligent Robots and Systems, 2011, pp. 1913-1918.

[25] S. Ozel, E. H. Skorina, M. Luo, W. Tao, F. Chen, Y. Pan, and C. D. Onal, "A composite soft bending actuation module with integrated curvature sensing," in 2016 IEEE International Conference on Robotics and Automation (ICRA), 2016, pp. 4963-4968.

[26] H. Zhao, R. Huang, and R. F. Shepherd, "Curvature control of soft orthotics via low cost solid-state optics," in 2016 IEEE International Conference on Robotics and Automation (ICRA), 2016, pp. 4008-4013.

[27] H. Zhao, K. O'Brien, S. Li, and R. F. Shepherd, "Optoelectronically innervated soft prosthetic hand via stretchable optical waveguides," Science Robotics, vol. 1, no. 1, 2016

[28] A. Koivikko, E. S. Raei, V. Sariola, M. Mosallaei, and M. Mantysalo, "Soft actuators with screen-printed curvature sensors," in 2017 IEEE SENSORS, 2017, pp. 1-3

[29] R. A. Bilodeau, E. L. White, and R. K. Kramer, "Monolithic fabrication of sensors and actuators in a soft robotic gripper," in 2015 IEEE/RSJ International Conference on Intelligent Robots and Systems (IROS), 2015, pp. 2324-2329.

[30] M. Giorelli, F. Renda, G. Ferri, and C. Laschi, "A feed-forward neural network learning the inverse kinetics of a soft cable-driven manipulator moving in three-dimensional space," in 2013 IEEE/RSJ International Conference on Intelligent Robots and Systems, 2013, pp. 5033-5039.

[31] M. Giorelli, F. Renda, M. Calisti, A. Arienti, G. Ferri, and C. Laschi, "Neural network and jacobian method for solving the inverse statics of a cable-driven soft arm with nonconstant curvature," IEEE Transactions on Robotics, vol. 31, no. 4, pp. 823-834, 2015.
[32] G. Runge, M. Wiese, and A. Raatz, "Fem-based training of artificial neural networks for modular soft robots," in 2017 IEEE International Conference on Robotics and Biomimetics (ROBIO), 2017, pp. 385-392.

[33] S. Han, T. Kim, D. Kim, Y. Park, and S. Jo, "Use of deep learning for characterization of microfluidic soft sensors," IEEE Robotics and Automation Letters, vol. 3, no. 2, pp. 873-880, 2018.

[34] I. M. Van Meerbeek, C. M. De Sa, and R. F. Shepherd, "Soft optoelectronic sensory foams with proprioception," Science Robotics, vol. 3, no. $24,2018$.

[35] T. G. Thuruthel, B. Shih, C. Laschi, and M. T. Tolley, "Soft robot perception using embedded soft sensors and recurrent neural networks," Science Robotics, vol. 4, no. 26, 2019.

[36] R. B. N. Scharff, R. M. Doornbusch, X. L. Klootwijk, A. A. Doshi, E. L. Doubrovski, J. Wu, J. M. P. Geraedts, and C. C. L. Wang, "Colorbased sensing of bending deformation on soft robots," in 2018 IEEE International Conference on Robotics and Automation (ICRA), 2018.

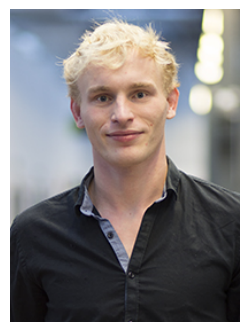

Rob B.N. Scharff received the M.Sc. degree in industrial design engineering from Delft University of Technology, Delft, the Netherlands, in 2015. He is currently working towards the Ph.D. degree. His research focuses on soft robotics, with an emphasis on the control of soft robots through embedded sensing and structural design.

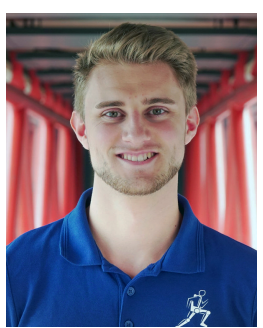

Rens M. Doornbusch received his BSc in industrial design engineering from Delft University of Technology, the Netherlands, in 2018. He is currently working as chief engineer of Project MARCH, a non-profit student-run organization. His work is focused on managing a team that is building a powered exoskeleton for paraplegics.

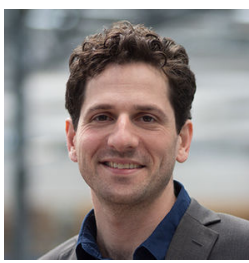

Eugeni L. Doubrovski Zjenja Doubrovski obtained his MSc degree in Integrated Product Design at the Delft University of Technology (TU Delft) in 2010. During his PhD project at the TU Delft, he worked on investigating new opportunities of Additive Manufacturing (AM) and developing new design methods for AM. After obtaining his $\mathrm{PhD}$ degree in 2016, he joined the TU Delft as assistant professor. His research interests include sustainability in 3D printing, developing and applying new mechatronic principles that are enabled by 3D printing, and new

3D printed material behaviour and appearance.

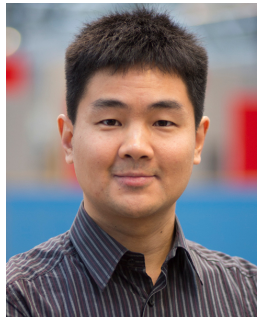

Jun $\mathbf{W u}$ is an assistant professor at the Department of Design Engineering, Delft University of Technology, the Netherlands. Before this, he was a Marie Curie postdoc fellow at the Department of Mechanical Engineering, Technical University of Denmark. He obtained a PhD in Computer Science in 2015 from TU Munich, Germany, and a PhD in Mechanical Engineering in 2012 from Beihang University, Beijing, China. His research is focused on computational design and digital fabrication, with an emphasis on topology optimization. 


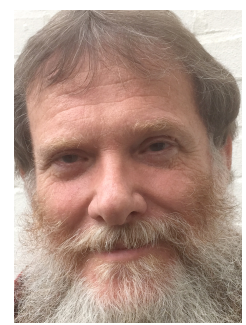

Jo M.P. Geraedts Prof. Jo Geraedts (1952) obtained a PhD in Physics at Radboud University, Nijmegen, the Netherlands. He joined Océ, a Canon group company, in 1983 and worked at the development of digital print processes and workflow for document and industrial printing. In 1997 Jo Geraedts became manager of the Océ Group Research department and was responsible for new technology developments. Research areas: mechatronics and modeling of print processes. From 2000 to 2013 he was manager of the Océ Industrial Design department and responsible for product, graphic, user interaction and usability design of all hardware and software developments in multidisciplinary teams worldwide. Jo Geraedts became in 2008 full Professor and chair Mechatronic Design at the faculty Industrial Design Engineering, Delft University of Technology, the Netherlands. His research focuses on 3D scanning, 3D multi-material printing, digital reproduction of Fine Arts, digital manufacturing and (soft) robotics.



Charlie C.L. Wang is currently a Professor of Mechanical and Automation Engineering and Director of Intelligent Design and Manufacturing Institute at the Chinese University of Hong Kong (CUHK). Before that, he was a tenured Professor and Chair of Advanced Manufacturing at Delft University of Technology (TU Delft), The Netherlands. He received the Ph.D. degree (2002) from Hong Kong University of Science and Technology in mechanical engineering, and is now a Fellow of the American Society of Mechanical Engineers (ASME) and the Hong Kong Institute of Engineers (HKIE). His research areas include geometric computing, intelligent design and advanced manufacturing. 